\title{
Erscheinung und Wesen.
}

Von Mroritis Šchiliclk, Rosțock.

Von der Philosophie habeñ îhe Freunde sowohl wie ihre "Gegner zuweilen behauptet,' daßß sie niemals in demselben Șinne "positive Arbèit leisten könne wie die Einzelwissenschaften. Sie bereichert, so heißt es, unser Wissen nie um neue Tatsachen, son derrn nimmt nur auf anderm Wege bereits gefundene Tatsachen aưf uh̀ di sucht sie in ihr System einzufügen. Man entgegnet wohl: die Tatsachen seien auch gar, nicht das Wichtige, einzig Belang'reiche und Wesentliche menschlicher Wissenschaft, sondern das" läge alleịn in den Gedanken über die Tatsachen, und in dem System dieser Gedanken, und deren Heimat sei in der Philosophie, von ihr wïrden sie in letzter Linie hervorgebracht.

Aber auch dies wird bestritten. Mit der Wahrnehmung der Tatsachen, sağt man, sind auch schon Gedanken über sie da, das Taktische ist nach Goethes Wort schon Theorie; das vorwissenschaftliche und das einzelwissenschaftliche Vorstellungsleben des Menschen ist schon durchsetzit von Begriffen, enthält einen Schatz von Begriffen; mit ihm arbeitet die Philosophie and muB mit ihm arbeiten, ohne ihn wahrhaft bereichern za können. Thre Anfgabe erschöpft 'sich nach dịesêr Meinang darin, diese Begriffe miteinander zu kombinieren, zu versöhnen and in ein widerspruchsloses System zu bringen. Herbart definierte die Philosophie als Bearbeitung der Begriffe, nicht etwa als Schöpfang der Begriffe. Das ist chärakteristisch. Er meinte, dâ alle vorphilosophischen Begriffe an Widersprüchen kranken, ùnd die Philosophie habe kein andres Zieil als diese Widersprüche zu überwinden, das wissensehaftliche Gedankensystem von ihnen .zu reinigen. Herbart war 
ein Freund der Philosophie, ihre Gegner aber éklärten es für einen Mangel, daß sie ihre letzten begrifflichen Elèmente nicht er:schaffe, sondern nur ordne and säuberè, sie sahen darin einen Beweis ihrer Frachtlosigkeit und Entbehrlichkeit und-priesen der. Philosophie gegenüber die Einzelwissenschaften als die allein schöpferischen; nur durch sie käme wahrhaft neues Material in die menschliche Erkenntnis and wirklicher Fortschritt.

Ich erinnere daran nicht, um in diesen Tadel mit einzustimmen, aber ich vertrete in der Tat die Meinung, daB" die - beschriebenen Anschauungen vom' W"esen der Philosophie einen richtigen und gesunden Kern enthalten, and sie sind nur insofern irrig, als sie damit zugleich ein Werturteil über die philosophische Arbeit fällen, mit dem sie ihr bitter unrecht tun und ihren Erfolg. verkennen..

Kant, in dessen Namen diese Studien erscheinen (womit bekanntlich nicht gesagt sein soll, daß ihre Mitarbeiter auf das Kंantsche System schwören, wohl aber, daß sie in ihm einen Lehrmeister der Philosophie sehen, irgendetwas an seiner Methode als vorbildlich bewundern), - Kant nannte seine Methode eine kritische. Er hat eingesehen, daß die Tat der Philosophie kritisch ist, nicht schöpferiseh in dem Sinne, wie die alte Metaphysik schöpferisch - sein wollte. Und wer die móderne Entwicklnng des wissenschaftlichen Denkens begreift, muß hier mit Kant übereinstimmen, and höchstens den Vorwurf werden wir zuweilen gegen ihn erheben, daß seine Methode noch nicht kritisch genug war.

Die geschilderten Auffassungen der Philosophie treffen aber nicht die Wahrheit, wenn sie von der richtigen Einsicht in ihren. kritischen Charakter za einer Geringschätzang philosophischen Strebens gelangen und seine Leistung für sekundär and für letzten Endes entbehrlich halten. Ganz das Gegenteil ist der Faill die kritische Arbeit krönt und vollendet erst die Einzelerkenntnisse, verbindét sie zar Einheit und zeigt ihren systematischen Zușammenhang. Vergegenwärtigen wir uns doch, was sie leistet: Kritische Reinigung und Versöhnnang der Begriffe, ihre Dúrçhbildang und Einfügung in ein System, das heißt logisch gesprochen weiter nichts als Entfernung jedes Widersprnchs aus den Urteilen, durch welche die Begriffe sich verknïpfen lassen. Ausmerzung aller Widersprüche, das bedentet aber: Befreinng von allen Irrtïmern! Jeder Irrtum nämlich muß sich zuletzt in irgendwelchen. Widersprüchen kundgeben, die er gebiert - denn wir verstehen eben nuter. Irrtum einen Gedanken, der irgendwie zom Widersprach 
mit der Erfahrung oder -mit bereits durch Deflnition feststehenden Begriffsbildungen fübrt. Alles Irrens aber ledig za sein - ein höheres Ziel gibt es für unser Forschen nicht. Vor dem Irrtam aber rettet man sich nur auf zwei Wegen: entweder man entsagt dem Denken überhaupt, oder man sucht das Heil in der Philosọphie.

Natürlich sind auch in vorphilosophischen Gedanjenbildunǵen schon Kräfte tätig, die an der Beseitigung der Widerspîn̈üche erfolgreich arbeitten, aber ihre Reiningung dringt nur bís zu gewissen allgemeinsten. Begriffen vor̃ die im Bètriebe des Lebens und der Einzel wisseñschafțen zünächst tunangefochteñ bleíben ḳönnen. Dièse höchsten Begriffe, diè wègen ihrêr Allgemeinheit alle andern

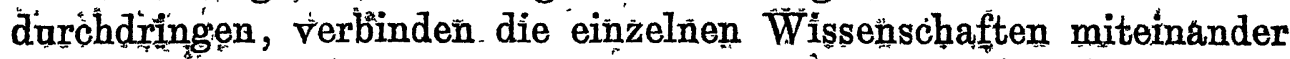

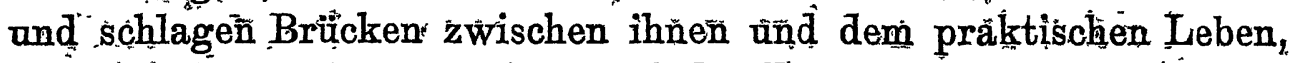

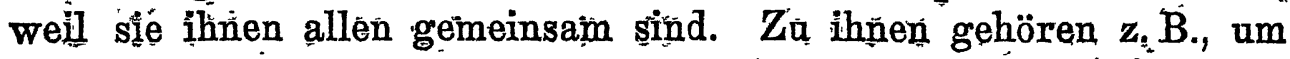
nur ein paar herausżugreifeñ, Raụn und Zeît, Ursáche, Stoff u. s: w. Sie bergen gerade die gröbte Zahl von Irrtumsmölichle sie am abstraktesten sind, sich am weitesten vom Greifbaren, Anschanlichen entfernen; und wenn es gilt, aus der Menge der über sie möglichen Urteile die widersprechenden auszuscheiden, so - ist das Sachè der allgemeinsten Wissenschaft, der Wissenschaft von den Prinzipien; der Philosophie, Wo auch die Einzelwissenschaften diesè Aufgábe direkt ángreifen (wie etwa die Physik es jetżt tut), da werden sie eben selber zur Philosophie. Ziwischen ihr und ihnen besteht :überhaupt nicht die scharfe Grenze, die man so oft vergeblich sucht, sie ist nicht eigentlich eine besondere Wissenschaft neben oder über ihren Schwestern, sondern sie ist etwas in ihnen, ihr Lebensprinzip; ihr treibender Geist; und der Philosoph ist nur dẹer Schatżgräber, der diesen Geist ans - Licht bringt.

So läßt șich das. Werk der Philosophie in der Tat auffassen als eine allmähliche Säuberàng menschlicher und wissenschaftlicher Meinungen von Irrtümern und Irrtumsmöglichkeiten. Sie schafft nicht unerhörte neue Wahrheiten, sondern scheidet ans dem. Wirrsal der Gedankien nach einer methodas exelusionis alles Falsche mehr ind mehr aus und reinigt es, wie Bacon die philosophische Aufgabe ganz richtig formulierte, von den idola d, $\mathrm{h}$ den Irrtimern, dén Widerspinichen, den Vorurteilen, in die der Mensch durch seine Geburt und Umgebung verstrickt ist. Räume alle Vorurteile weg, and du bebältšst die Wahrheit rein übrig, denn jedes falsche Ürteil maß ein Vorurteil, ein vorschnell gefaßtes Urteil, sein.

Werin es solehergestalt die Aufgabe der Phílosophie ausmacht, 
fundamentale Widersprüche zu überwinden; so müssen ihré großen Probleme aus gewissen Unverträglichkeiten entspringen, aus gewissen Begriffsgégensätzen, die zur Quelle von Widersprüchen werden. Und so verhält es sich wirklich: alle großen philosophischen Fragen werden am deutlichsten durch ein gegensätzliches Begriffspaar bezeichnet. Ich branche nur einige soleher Paare zu nennen, damit wir die Richtigkeit dieser Behauptüng einsehen: Freiheit and Notwendigkeit, Leib and Seele, Egoismus and Altraismas, Subjekt und Óbjekt, Erscheinang und Wesen. Die Schwî̀rigkeiten der durch diese Begriffspaare gekennzeichneten Probleme, beruhen tatsächlich - was nicht immer richtig erkannt wird auf gewissen direkten Widersprüchen, zu denen sie überaus leicht Anlaß geben - aber ich muß mir hier versagen, das näher nachzúweisen, und statt dessen lièber von den Möglichkeitẹn reden,. diese Widersprüche aufzulösen.

Die großen Bègriffsgègensätze der Philosophie, von deneñ ich soeben einige aufgezăhlt habe, sịnd nưn keinèswegs unabhängig voneinander, sondern sie hängen auf eigentümliche Weise zũsammen, so dab man fast sagen kann: alle diese Probleme verlieren auf einmal ihren Schrecken, wenn man nur eins von ihnen restlos gelöst hat. Wenn wir uns daher'nunmehr dem Begriffspaar Wesen und Erscheinung zuwenden, so werden sich ganz von selbst, und ohne daß ich darauf' aufmerksam mache, manche Beziehungen zeigen. etwa za der psychophysischen Frage oder zu dem Spbjekt-ObjektProblem -, Beziehangen, die hier nicht weiter verfolgt werden können.

Die fraglichen Begriffspaare führen zu Widersprüchen w wil sie falsch gebildet sind. Sie bilden so lange ein Problem, als sie die Träger gewisser Vorurteile bleiben, und es ist gelöst, wenn diese unbegrïndeten Urteile als Quelle aufgedeckt sind. Wie eigentlich der Irrtum überhaupt zastande kommt, worauf seine Möglichkeit beruht, ist eine noch wenig behandelte Frage, für den Philosophen wohl nicht weniger interessant, als es die Frage nach dem Ursprang des Bösen für den Theologen sein mag.

Worin also liegt der Grund, der Rechtsgrand für die Aufstellung des Gegensatzes von Wesen und Erschein ung? Man wird ihn suchen müssen in dem Unterschied des Konstanten und V a riablen, des Bleibenden und des Flüchtigen in unserm Erleben; vor allem den sinnlichen Wahrnehmungen. Sie sind fließend, ungleich and veränderlich. Ein Haas sieht grundverschieden aus, je nach-

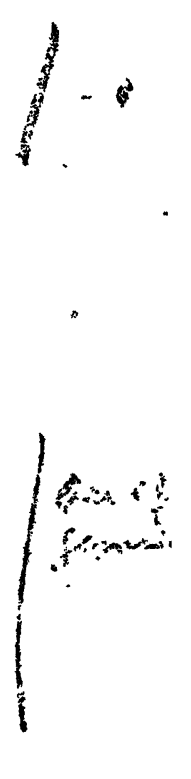


dem ich es am Morgen oder am Abènd, von rechts oder von links, von innen oder von außen betrachte. Und doch ist es dasselbe Haus; wìrd dūrch denselben Nameñ, denselben Begriff bezeichnet, es ist irgend etwas an ihm, das sich nicht verändert, und irgendwie mab man doch wohl auf dieses Konstante zielen, wenn man dass Wort "Wesen" gebrạucht, und auf jène flüchtigen Einzelwahrnebunungen,"-wenn man voń "Erscheinangen" špricht. Das Erkiennen ñämlich richtetet sich stets auf das Unveränderlichè, Gleichbleibende, denn Erkenntnis ist überhaupt gar nichts anderes als die Ánffindüing des Glèichehen in dem Verschiedenen. So bedeutet W. es en den ruhenden Pol in der Fîncht der Ersche inungen. Diese Gegentiberstêling "des Konstanten, Identischen nid des Fließenden, Verschiedenen ist sicherlich berechtigt and von fundamentaler Bedeutung. Solange die Trennang von Wesen and Erschênung nichts weiter bedeuten möchte als dies, muß sie wider sprachslos' durchfuhrobar sein. Aber schon die "Wortbildung deatet $a \grave{n}$, dẩ eben noch etwàs anderes gemeint ist, daß man in dieses Begriffspāär noch viel mehr hineingelegt hat.' Und in diesem Mehr liẹgen tausend̆ Irrtumsmöglichkeiten. Kaum ist jener Gegensatz aufgestellt, so hängen sich schon Nebengedanken an ihn, die den Widerspruch fäst unvermeidlich machen. Der ältestè Fehler besteht darin, dảb man vergißt, zwischen Erscheinung and Schein za unterscheiden; ihn haben bereits die Eleaten begangen, bei denen überhanpt das Begriffspaar, von dem wir sprechen, seine erste Ausprägung findet. Schein aber bedentet das Unwirkliche, und wèn Erscheinung damit identifiziert wird, so wird zugleich das Wesen mit dem Wirklichen identisch. Das Wesen also, d. h. der Gegenstand der Erkenntnis, der Inhalt des Denkens, der Inhalt der Begriffe, wird zum wahren Sein: das Denken ist dasselbe wie das Sein, so formulierten es die Eleaten. Die Erscheinung aber, der Inhalt der Wahrnehmung im Gegensatz zam $W^{7}$ esen als Inhalt des Denkens, wird zum Nichtseienden, zam Trug, zur Täuschung. Die Wirklichkeit der Wahrnehmungswelt wird einfach gelengnet. Von dem Wesen der Welt, das bei den Eleaten als reines, nnveränderliches Sein in erhabener, aber glanzloser Starrheit dasteht, weiß $P$ laton ein strahlendies Bild hinreißend za entwerfen. Ihm offenbart sich das wahre Sein als Reich der Ideen: die Ideen, d. h. die Begriffe, die Denkinhalte, das Gedachte, sind das

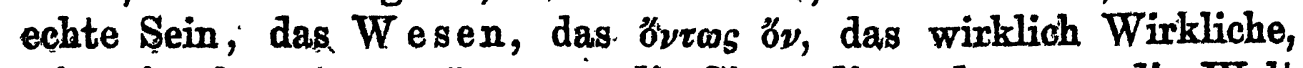
wie wir übersetzen müssen - die Sinnendinge dagegen, die Welt 
der Wahrnehmungen, Erde, Flüssee, Berge, Bäume, sie sind nicht in demselben Sinne wirklich, nicht Wesen. - Natürlich wird man der Platonischen Philosophie nicht gerecht, wenn man einfach den Gegensatz Iileenwelt-Sinnenwelt mit dem Gegensatz Wesen ' Erscheinung gleichsetzt; viel mehr, viel tieferes liegt noch darin aber dies Verhältnis Wesen-Erscheinang liegt unzwíneifelhaft a u cb darin. Nicht mehr, wie für die Eleaten, sind für Platon die Sinnendinge nur Schein, damit lasśen sie sich nicht abtan," sie sind. nicht wesen Io s, sondern haben eben doch teil an den Ideen, am Wesen. Die unsägliche Mühe, mit der Platon dies Thilhabe der Einzeldinge an den Ideen zu erklären and zu rechtfertigen sucht, ohne daß es ihm doch gelänge, das ist eben die Mühe des Kampfes mit dem Problem Wesen-Erscheinung, wie er ganz ähnlich bis in nnsere Zeit hinein noch gekämpft, wird. Es ist das Problem, das immer da entstehen maß, wo man arivei verschiedene Arten der Wirklichkeit unterscheidet, eine wesenhafte und eine erscheinungshafte, eine subjektive and eine objektive, eine psychische and eine physische, eine substantielle und eine akzidentielle, - oder wie die Gegensätze sonst noch heißen mögen. Die Eleaten wollten das Problem aus der Welt schaffen, indem sie den sinnlichen Gegenständen die Teilnahme am Reiche der Wirklichkeit einfach verwehrten; Platon mußte der Wahrnehmungswelt ihr Recht lassen, and er versuchte es, indem er - wenn auch nicht in dieser grobschlächtigen Formulierang - ihre Wirklichkeit als eine andere, eine geringere, von der eigentlichen kernhaften Realität der Ideen unterschied. Und" damit hat der Gegensatz von Wesen und Erscheinung bei ihm einen Gehalt gewonnen, welcher heute noch einên wichtigen Teil der Bedentang des Begriffspaares ansmacht. Denn wer Erscheinung und Wesen einander gegenüberstellt, der denkt. dabei - mag es ihm ausdrücklich zum Bewaßtsein kommen oder nicht - an zwei verschiedene Gra de der Wirklichkeit: das Wesen ist die echte, die Erscheinang eine sekundäre Art der Realität.

Diese Unterscheidang zweier verschiedener Seinswesen ist, wie gesagt, der zentrale Punkt. Wir werden in ihr das jo erkennen, das Anlaß gibt zu dem Problem, und damit za einer Kette von Problemen, an der durchans die schwersten philosophischen Entscheidangen hängen. Man kommt, behaupte ịch, ihrer Lösung ein gates Stück näher, wenn man den Gedanken mehrerer Seinsweisen, verschiedener Realitätsgrade seinerseits uberwintet - oder besser noch, ihn ron vornherein vërmeidet. Dann töonninen 
jene Probleme sich überhaupt nicht voll entfaltén, sie werden als fehlerhafte Fragestellungen erkannt. - Damit wir uns aber an dem Siege über den Irrtum so recht erfreuen können, wollen wir uns einen Augenblick in dem Gebiete uimtan, in dem er herrscht, and zusehen, welche Unordnung er dort angerichtet hat.

Überall, wo verschiedene Arten der Wirklichkeit unterschieden werden, eine wesenhafte und eine erscheinungshafte - wenn nicht gar noch mehr Arten daneben - wird auch alsbald eine Stafenfolge und eine Rangordnung zwischen ihnen behauptet. Die eine Art des -Seins gilt für höher, echtep, vornehmer, wichtiger als die andere, d. h, es spielt der Wertgedanke hier hinein. Gerade bei Piaton ist diese Verweehslung oder "Identifizierung des Wertgeșichlíspuñultes mit dem logischen Gesichtspunkte am offenkundigsten. Dieseri Platonismus ist auch heut noch unter uns auf die verschiedenste Weise wirksam; er ist im Grunde daran schuld, da $B$ das Wort Idealismus so oft inißbraucht wird und rein 'sachliche Beưrteiluñgen dür̆ch ethische, aesthetische und religiöse Wertschätzungen veriwiirrt und getrübt werden. Doch diese Verzweigungen der Gegenäätze können hier nicht weiter verfolgt werden; sie bildèn ein besondệres Kapitel für sich. - Als die höhere, bedeutsamere, wer̂troHere Wrirklichkeit gilt dann immer die fernere, schwerer zu erreichende, also in unserm Falle diejenige des Wesen's gegenüber der des Erscheinens. Das ist verständlich. Denn das Fremde, Entférnte weckt eigenartige, stärkere Gefühlsbetonungen als das Nahe, Alltägliche; die nur dem Denken erreich- bare Realität wird daher für erhabener geschätzt als die unmittelbar und mühelos gegebene, wahrgenommene. Als unmittelbar gegeben galten aber dem antiken Denken die physischen Objèkte selber, aus denen ${ }^{-}$die in heraklitischem Fließen begriffene Sinnenwelt sich aufbaut. Nicht sie sind daher das wahrhaft WirkTiche, sondern die allem heraklitischen Fließẹn entzogenen, über die Sinnenwelt' erhabenen Ideen, za denen das Denken erst durch ñühisame Arbeit vordringt, die aber in den Wahrnehmungsgegenständen sich offenbaren.

Weñn nun auch in der Folgezeit die Begriffe von Wesen und Frscheinung einen etwas anderen Inhalt gewannen, so blieb doch ihr gegenseitiges Vérhältnis ím groBen Ganzen dasselbe: sie rücken gleichsam beide dem Menschen näher, ohne doch ihren gegenseitigen Ábstand zu ändern. Das geschah besonders durch die Entdeckung dèr Subjektivität der Sinnesqualitäten durch Demokrit, den älteren 
Zeitgenossen Platons. Damit wird das Problem eigentlich bereits vor Plato auf die Ebene gehoben, auf der es sich in dèr neuerèn Philosophie bewegt; es tritt nämlich dadurch ' ein Moment hinzu, das in der neueren Auffassung einen integrierenden Bestandteil des Erscheinungsbegriffes bildet und ihn erst vollständig machìt: eben das Moment der Subjektivität. Zur Eigentümlichkeit der Erscheinang gehört erstens ein Objekt, dàs da erscheint, und ein Subjekt, dem es erscheint. Erscheinung ist stets Erscheinung v on etwas and Erscheinung für jemand. Das Wesen dagegen ist an sich und für sich selbst. Das Wesen, das wirklich Wirkliche, sind bei Demokrit die Atome, also die phýsischen Objekte. Ihr Wesen erfassen wir nach seiner Erklärung allein durch das Denken; die sèkandäre Wirklichkeit aber erblickt er in den Sinnesqualitäten, den Empfindungsinhalten rot, grün, warm, kalt, süß, bitter u.s. w., in ihnen erkennt er das unmittelbar Gegebene, und er verachtet sie, weil sie die Bedingung der Konstanz, der Identität nicht erfüllen, ohne welche, wie wir gesehen haben, das Denken nicht zum Erkennen wird. Demokrits Standpunkt ist in unserer Frage derselbe wie derjenige des Materialismus des vorigen Jahrhunderts, aach die Materialisten ${ }_{\oplus}$ vergaßen in ihrer Bewunderung der Realität des physischen Stoffes vollkommen, dab es auch eine Welt der Bewaßtseinswirklichkeit gibt - oder 'glaubten doch so sprechen za dürfen, als wenn sie überhaupt gar nicht da wäre. Die Erscheinung hat also hier, fast wie bei den Eleaten, eine so sekundäre Wirklichkeit, daß sie wiederam einfach zum Nichts wird.

Ein solcher Standpunkt ist unmöglich in jedem erkenntnistheoretisch fundierten System der neueren Philosophie. Denn die neuere Philosophie beginnt, als Descartes den Satz an die Spitze stellt: Gerade das unmittelbar gegebene, die Bewaßtseinswirklichkeit, das Erlebnis - oder wie man es nun bezeichnen will -, gerade dies ist schlechthin wirklich, besitzt echte, vollgültige Realität, nnd wenn auch außer ihr überhaupt nichts anderes real wäre. Dieser Satz von der Realität der BewuBtseinsinhalte - gewöhnlich in die Formel "cogito ergo sum" gekleidet, und bekanntlich schon vor Descartes gelegentlich ausgesprochen - bedentet nicht etwa die Auffindung einer besondèren, vorher unbekannten Eigentümlichkeit der BewuBtseinsdaten, nämlich ihrer Existenz, ihrer nnumstöBlichen Wirklichkeit, sondern er bedeutet vielmehr umgekehrt die Anfdeckung des reinen Sinnes und der wahren Quelle des Wirklichkeitsbegriffes. Wir nennen eben das wirklich, 
was wir unmittelbar erleben Nicht das. Denken, sondern in Ietzter Linie nur das Erlebnis lehrt uns, was unbezweifelbar und schlechthin virkich ist. Was unter Realität zu verstehen ist, läBt sich nicht definieren, sondern nur aufzeigen, ebenso wie man nicht - - definieren kann, was "Lust" ist, oder was "rot" ist. Der. Erlebnisinhalt ist der Prototyp alles Realen, ganz allein in ihm liegt die Wurzel des Wirklichkeitsbegriffs; und wo unser Denken dar̃über hinaús ein Sein als wirklich annimmt, dás nicht Bewửitseîn ist, àlsọ ein trañszendentes Sein, da bedarf es dazu doch îngend wellcher Hinwịise und Anhaltspunkte im unmittelbar Gegebenen; von diesèm, vöm Eriebnis geht also letztlich alile Realitätssetzung aus.

Nảch dẹ Descarteșschen Klärung dieses Sachverhalts wird von nun ân făst immer dạs Eirlebte, das Subjektive, das Psychische als' echtes Wesen anerkannt; und wer sich mit den Psychologie der metaphysischeñ Ŝsytème beschäftigt hat, wandert sich nicht darüber, dàß nư bald der Sp̈pieß noch ein wenig weiter herumgedreht wurde und jetzt gerade das objektive; außerbewubte Sein, früher als das eigentliche $W$ esen geachtet, zur Realität zweiten Grades herabgewürdigt warde. Ziwar zur Erscheinung konnte es. nicht wohl gemacht -werden, dazu fehlte ihm das Merkmal der Súbjektivität; es mußte für Trug and Scchein, oder für ein bloßes Nịchts, höchstens für eine Hilfskonstrüktion erklärt werden. In der Taut finden wir diesen Standpunkt auch oft genug aúsgebildet: es ist der des-subjektiven Idealismus, wie z. B. Berkeley ihn vertreten hat. Thim ist das objektive Sein, das Physische, die Materie, überhaupt nịcht wirklich. Realität kommt einzig dem Psychischen, den BewaBtseinsinhalten za, die irgend welchen Subjekten gegeben sein müssen, und wo Eirzelindividuen als Subjekte nicht in Frage kommen, da tritt an íbre Stelle das allumfassende Sabjekt, nämlich Gott. Hier hat also der Dualismus von Wesen und Erscheinung bereits' einem metaphysischen Monismus Platz gemacht. Auch moderne Richtungen passen durchaus in den Rahmen dieser.Anschaunngen hinein, so der Psychomonismus, wie er etwa von dem holländischen Philosophen Heymans and manchen andern verteid̈igt wird.

Aber bekanintlich hat die Descartessche Wendung eine solche Wirkang nur vereinzelt entfaltet - von vielen Denkern wurde der Gegensatz von Erscheinung und Wesen anch fernerhin beibehalten, ja noch schärfer und deutlicher ausgeprägt. Es bleibt ihnen 
vor allem häufig noch eine Neigung, die Wirklichkeit des Erlebens herabzusetzen zugunsten des Transzendenten, und diesem Hang begegnet man auch bei solchen Philosophen, die bewußt von der ursprünglichsten Realität der Bewrobtseinsinhalte ausgehen und sich bemïhen, ihr volles Recht widerfahren zu lassen.

So vor allen bei Kant. Der eben beschriebene Hang bricht in seiner praktischen Philosophie mit Gewalt hervor; aber auch in der Erkenntnistheorie s7eist er dem Sein des Nichtgegebenen, des Nichterlebten einen ausgezeichneten Platz an vor der Wirklichkeit des Erlebens. 'Das erstere ist ihm das Reich der Dinge an sich, das letztere bezeichnet er als Erscheinung. Der Begriffdes Dinges an sich vertritt bei Kant in der Tat genau das, was wir bisher als "Wesen" bezeichneten, denn die Dinge an sich sind das aller Subjektivitäät Entrückte. Er wird nicht müde, die nloßen Erscheinungen" und die "selbständigen Wesen" einander gegenüberzustellen ${ }^{1}$ ). Natürlich sind die Erscheinungen für Kant etwas Reales; immer wieder hat er ja betont, daß Erscheinung nicht za verwechseln sei mit Schein. Die sinnliche Körperwelt hat aúch bei Kant diejenige volle Realität und Objektivität, mit der sie jedermann in Leben und Wissensehaft gegenübertritt, aber Kant unterscheidet doch ihre Realität als eine empirische von der „ab= soluten" der Dinge an sich. Dies seine eigene Ausdrucksweise. So kann es nicht ausbleiben, daß das Sein der Dinge an sich als ein echteres, kernhafteres gewertet wird: die Welt der Naturdinge ist ${ }_{n}$ nar ${ }^{\prime}$ Erscheinung. Der Begriff des Phänomens setzt etwas voraus, das selbst nicht Phänomen ist, sondern eben mehr als Erscheinnng. In dem "nur" and dem „bloß", mit dem Kant von den Erscheinungen redet, offenbart sich immer wieder der Gedanke, als komme den Dingen an sich eine "höhere" Realität zu. Die Erscheinungen sind, wie er stets wiederholt, bloße Vorstellungen des Gemüts, d. h.; Inhalte unseres Bewabtseins. Mithin sind die Dinge an sich Wirklichkeiten, die nicht nur im Bewußtsein, sondern als selbständige Wesen für sich existieren, absolut, nicht einem Subjekt gegeben sind, sondern den Vorstellungen des Subjekts ${ }_{n}$ zagrunde liegen ${ }^{\prime}$, in ihnen erscheinen. So treten das im Bewratsein gegebene and das nicht so gegebene Sein als zwei verschiedene Arten der Realität auseinander: die Dinge können wohl da sein, ohne za erscheinen, aber die Erscheinangen können

-1) z. B. Kritik der reinen Vernunft, Ausgabe Kehrbach, S. 314 f. 
nicht da sein ohne Dinge, ibr Sein ist diesen gegenüber ein nnselbständiges, abhängiges, sekundäṛ̣̂es. Moderne Denker haben es auch in der Terminologie zum deutlichen Ausdruck bringen wollen, $\mathrm{daB}$ sie hier tatsächlich einen Ưnters schied der Realität selber vorliegend erachten: so verwandte Ku $\mathrm{i} I$ pe das Wort "wirklich" nur für das anmittelbar Gegebene, und bezog das Wort "real" nur auf die bewaßtseinstranszendènte Welt. Er sagt von den „wirklichen" und den "realen" Elementen, dẩ zwischen ịnen eine "nähe Beziehung " bestehe - aber dàit ist natürlich das Verhältnis zwischen den beiden Reichen eher verdunkelt als geklärt. Un Und selbst wenn mañ den Gedanken abwehrt, die Realität des einen von ihnen für echter, höher anzusehen âls die andere, so bleibt doch ihre bloße. Enterscheidung äls ein Fehler bestehen, dem unlösbare Probleme entquellen.

Die Kantsche Philosophie schafft an diesér Stelle keine größere Klarheit als die Platonische, welchê die Art des Teilhabens der Dinge an den Ideen nicht plausibel machen konnte. Wenn man mit Kant eine Vorstellung ( $d \cdot h$. eine Währnehmungsvorstellung) als die Erscheinung eines Dinges bezeichnet, so meint man natïrlich nicht, $d a B$ sie etwa ein Teil des Dinges sei, ein Ausflub, der in dạs Bew̧ußtsein hineinströme od̀er hineinrage. Überflüssig, zu sagen, daß die Erscheinüng auch nicht etwa als ein. Bildz des erscheinenden Dinges anfgefaßt werden darf. Solche naiven Denkweisen sind ja in der nachkantischen Philosophie mit Recht kaum wieder im Ernst aufgetreten. Und wenn man, statt von einem Bilde ža s̀jpechen, sie als Projektion, Nachahmung, Abschattring oder sonstwie bezeichnen wollte, so ist damit natürlich außer der Einführang neuer Worte nichts weiter gewonnen. Tatsächlich ist mit der Untèrscheidung dieser beiden Sphären des Seins ein besonderes Verhältnis statuiert, das ssich gar nicht weiter klären läBt, weil es einzigartige ist, nicht auf ein anderes bekanntes zurückgefüuhrt werden kanñ, Es ließe sich höchstens durch Analogien verdeutlichen, die der empirischen Welt - also, in der Sprechweise des Systems, der Welt der Erscheinungen entnommen sind. Ein solcher Verdeutlichungsviersuch soll es offenbar sein, wenn Kant, die Erscheinungen als die Wirkungen bezeichnet, welche die Dinge an sich anf das Bewrütsein aúsüben, wồrtlich: als „Vorstellungen, die sie in uns wirken, indem sie unsere Sinne: affiżieren". Das muB, wie gesagt, bildlich gemeint sein, denn wir wissen, daß nach Kantscher Lehre die Begriffe Ursache and Wir- 
kang nur im Reich der Erscheinungen Sinn und Bedeutang haben und daher nicht auf ihr Verhältnis zum Reiche des Wesens angewandt werden dürfen. Daß Kant dies scheinbar doch tat, hat ihm ja bekanntlich schon von den Zeitgenossen den Vorwarf des Selbstwiderspruchs eingetragen,

Nun könnte man ja bei dem Daalismus stehen bleiben, ihn als unvermeidliches Übel hinnehmen, man kann sagen: es is t ebèn so, daß Dinge einerseits and Erscheinungen andrerseits existieren and in eirem nicht weiter a aufzạklärenden Verhältnins żueinander stehen. Aber erstens wäre es unbefriedigend, die zwei Reiche getrennt und unredužierbar nebeneinander anżunehmen, denn der Wille zar Erkenntnis fordert die Reduktion des einen auf das -andere - Erkenntnis ist nichts anderes áls solche Reduktion and deshalb kann das Denken sich bei einem derartigen Duälismus nicht beruhigen; zweitens aber, and das ist entscheidend, stellt sich anch die Kantsche Fassung unseres Gegensatzes überhaupt. als undurchführbar heraus, weil sie im Rahmen seines Systems an innerem Zwiespalt, an Widersprụ̈chen leidet. Nicht $z w a r$, daß jeder Begriff eines Dinges an sich notwendig nnvollziehbar wäre - davon kann ich mich durchans nicht überzengen -, aber. er. wird überflüssig und vollkommen bedeutungslos, man verliert jedes: Recht, das Dasein von Dingen an sich noch zu behaupten, wenn man mit Kant jede angebbare Beziehung der Erscheinungen zu ihnen leugnet. Unter der Voraussetzung einer solchen Beziehungslosiǵkeit nämlich kann in den Eirscheinungen - und außer ihnen ist uns ja nichts bekannt and gegeben - niemals ein Hinweis auf die Existenz von transzendenten Dingen liegen, denn jeder Hinweis setzt irgend eine angebbare Beziehung voraus, er bedeutet. ja gerade eine solche, besteht in nichts anderem. Deshalb würde jeder Grand fehlen, ein von der Sphäre der Erscheinungen unterschiedenes Reich des Wesens überhaupt anzunehmen. Man kann nicht das Dasein vion etwas behaupten, ohne zu wissen, wovon man denn das Dasein behauptet. Es gịbt kein Wissen über Existenz ohne jedes Wissen über Essenz. Hieran muB jeder strenge Phänomenalismas in letzter Linie scheitern. Opfert man aber dieDinge an sich, so stellt man sich damit - das kann modernen Deatungsversuchen gegenüber nicht genug herviorgehoben werden gänzlich außerhalb der Kantschen Philosophie. Ohne das Ding an: sich kann man nach dem bekannten Wort Jakobis tatsächlich nicht. in das Kantische System hineingelangen. 
Die zweite Möglichkeit wäre, den Gedanken der gänzlichen Beziehungslosigkeit beider Reiche fallen zu lassen; auch damit aber wäre ein bedentsamer, charakteristischer Zug in dem Kritizismus Kants verwischt; die Unnahbarkeit, Unerreichbarkeit des Wesens waäre dahin, die Dinge wären erkẹnnbar geworden und damit ein ganz prinzipieller Unterschied aufgehoben, der bei Kant Erscheinūng and Wesen voñeinander trennt, denn er fällt für ihn zusammen mit dem Gegensațz der unerkennbaren. ùnd der erkennbaren Wirlelichkeit. Immerhin drängen die im Kritizismus liegenden Kème viel mehr žn einer Entwicklung in Richțung der zweiten als in Richtung dëŕ ersten Möglichkeit, die etwa auf Fichtesche Wege führt. Ich komme sogleich daraúf zurück.

Wir sẹhen einstweilen; daß es anch Kant nicht gelingt, das Begriffspaär Wesen=Erscheinung in befriedigenderer Weise zu fassen als seine Vorgänger; die sich, mit dieser Dualität abmühten. Die Schwierigkeiten würden sich 'noch deatlicher' zeigen und ihren Charakter als unaưflösliche Widersprüche offenbaren, wenn wir die Beziehung unseres Problems zum psychophysischen Problem verfolgen wollten, das auf' engste mit ihm verknüpft ist und auch Jei Kant so verkhïupft erscheint, oder wenn wir gar auf die Lehre vom sog. innern Sin̄n eingehen würden, durch welche Kant den Gegénsatz von Wesen und Erscheinung in ein Gebiet za tragen suchte, das bis dahin noch von ihm verschont geblieben war und nur durch ganz-künstliche Maßnahmen unter seine Herrschaft gebracht werden konnte. Aber ich muB auf diese Prüfung: hier verzichten, denn es ist an der Zeit; endlich die systematische Hauptfrage zu stellen, wie dér Knoten denn nun zu lösen sei, der unweigerlich durch die Annahme zwieier Arten von Realität geschürzt wird.

Die nächstliegende Lösung würde natürlich darin bestehen, eine der beiden Arten gänzlich zu streichen, schlechthin für nicht vorhanden zù erklären. Im Altertum strichen die Eleaten das Reich der Eischeinungen aus der Wirklichkeit weg - in unserer Periode der neueren Philosophie aber kann nieht der geringste Zweifel sein, daß höchstens der umgekehrte Versuch gemacht werden könnte: soll eine Realität aus dem metaphysischen Weltbild ausgemerzt werden, so kann es nur die der Dinge an sich sein, denn die sog. Erscheinungen, das Gegebere, die Bewaßtseinsinhalte, stehen jenseits aller philosophischen Künste. Sie sind schlechthin wirklich. Sie sind die Elemente, aus denen die Welt 
der Phänomene sich aufbaut. Daß sie die einzige Realität-darstellen, ist die Behauptang des strengen Positivismus.- Dié Elemente des Bewaßtseinslebens - rot, grün, warm, kalt, süß; bitter etc., sind als unmittelbar gegeben einfach real, und der $\mathrm{Po}$ sitivismus fügt nun hinzu, daß außer ihneñ und ihren Beziehangen zueinander nichts andres real ist, dab es neben ihnen nichts anderes gibt. Dieser Standpunkt, dessen bedeutendster Vertreter in der Gegenwart Mach gewes̀en ist, setzt also Wirklichlkeit iden. tisch mit unmittelbarer Gegebenheit. -

Das ist aber dann eine blobe $e_{*}$ Definition des Realitätsbegriffs; nnd als solche willkürlich, ohne gehörige Rücksicht anf die Funktion dieses Begriffes in Wissenschaft und Leben aufgestellt. Es bedentet ihnen gegenüber eine-Einengang der Bedeatung.des. Wortes .n wirklich", die das Realitätsproblem durch eine Definition abschneidet, statt es zn lösen, denn dieses Problem stellt gerade die Frage, ob etwa noch anderes als das schlechthin Gegebene als" wirklich za bezeichnen sei. Auf diese Weise gerät die positivistische Auffassung in.Schwierigkeiten, wenn sie von dem echten Sinn der Realwissenschaften Rechenschaft geben will - doch ich kann das hier nicht näher begründen und will mich sogleich zar Besprechung der übrigen Móglichkêiten wenden, durch die man versuchen kann, des Gegensatzes Wesen-Erscheinung als zweier vẹrschiedener Realitätsstufen Herr zu werden.

Der. strenge Positivismus wollte es erreichen, indem er der einen von beiden überhaupt die Anerkennung versagte, er strich sie fort und behielt allein diejenige Sphäre übrig, welche Kant Erscheinung nannte, und dadurch warde sie zum. Wesen erhöht. Man kann den Zwiespalt aber auch dadurch zu überwinden suchen, daß man ihn auffabt nicht als eine Abgrenzang zweier Wirklichkeitssphären, sondern blob als eine logische oder methodische Unterscheidang. Dies ist der Standpankt der neakantischen Richtangen der Gegenwart, von denen die einen glauben, damit die wahre Kantsche Meinung za treffen, während die andern eine bewaBte Weiterbildang and Vollendang seiner Gedanken zu geben meinen. Auch für diese Ansicht gibt es. nur eine Wirklichkeit, and in ihr wird der Gegensatz zwischen Subjekt and Objekt, 2wischen Sein and Erscheinen darch das Denken erst geschaffen, ja die Wirklichkeit wird als solche selbst erst durch das Denken erzeugt, zar Wirklichkeit gemacht darch den Richterspruch der Yernunft - im Gegensatz zax positivistischen Anschanung, nach 
welcher die Realität vor állem Denken gegeben ist. Realität, so sagt man nämilich, ist ja eine Kategorie, ein Verstandesbegriff, eine Denkform, in der wir Gegenstände denken; es ist also der Verstand, das 'Denken, welches die Gegenstände zum Range von Realitäten 'erhebt, indem es eben dỉe Kategorie der Realität anf sie anwendet - sie habonen daher̀ keinè Wirklichkeit nnabhängig vom Denken. Die Behauptang einer solchen Unabhängigkeit wärein 'sich widersprechend, sinnlos, weil Realität eben selber eine logische Form ist. M. a. W.: ein unabhängiges Wesen, Dinge an sich, gibt es nicht. 'Sie dienen höchstens als methodiseher Grenzbègṛiff, wie die Unterșcheídung von Subjektivität und Objektivität in ihren vierschiedenen Stafẻn gleichfalls nur methodische Bedentüng hat. Die im wàchen Leben wahrgenommenen Gegenstände sìnd objẹktiv gegenüber den Traumgestalten, aber subjèktiv gegenüber den durch die mathematische Naturwissenschaft bestimmten: Gëgenständen: Diese Únterscheidungen sind lorrelativ zueinander; Subjektivität nnd Objektivität bezeichnen keine absoluten Gegenš̃útze; sondern verschiedene Erkenntnisstufen, und das Begriffspaar "Wesén-Erscheinung, in dem dieser Gegensatz als eine absoluter aúftritt, kann ửberhậpt keiǹe Añwendung mehr finden, und dieser logische I deàli s.mu ư's erklärt ihn daher für restlos überwanden:

Gegen die darges̃tellte L̇ehre ist einzawenden, was überhaupt grgen jeden Rationalismus eingewandt werden muB. vergeblich iste das Béminhen, das Sein durch das Denken za begründen, reale Béziehungen auf logisehe zurückzuführen. "Dies gêlingit dèn logi"sçhẹñ İdeälismus der Nenkantianer so wenig, wie es Hegel geliungen ist. Es bleibt ein bedeatungsloses- Spiel mit Gedanken fäst möchte man sagen, mit Worten, wenn das Denken, das Logische, dem Sein, dem Wirklichen übergeordnet wird, wenn man beides identifiziert, weil doch alles Sein schließlich Denkinhalt sei. In Wahrheit ist Realität metwas Irationales; deatlicher ansgêrückt: der Wirklichkeitsbegxiff ist undefinierbar; seinen Inhalt durch Begriffe zu bestimmen, ist unimöglich. Was „wirklich" heißt, läßt sich nicht logisch erklären, nicht auf étwas zurückführen, daś nochh nicht wirklich wäre, sondern es läBt sich nur im Erleben ấñûzejgen. Ich wies, schon vorhin darauf hin, was ja auch von der Mehrzahl der Erkenntnistheoretiker anerkannt wird, daß der Begriff des Wirklichèi seine letzte, Quelle im anmittelbar, Gegebenen hat, den Bewußitseinsdaten, den Empfindangen und Gefühlen. Derr Gedanke, daš Logische, kañn nicht zum -Richter über diese 
Wirklichkeit gemacht werden, sondern setzt sie jederzeit vorans Dies war gerade auch fïr Kant selbstverständlich: er hat besonderș erklärt, daß bei der Descartesschen Fráge näch der Realität-der Bewaßtseinsinhalte die Existen z noch Keine Kategorie sei, und damit die vorlogische Realität anerkannt. Deshalb ist der eben skizzierte logische Idealismus nicht die natürliche Fortsetzung der Kantschen Philosophie, sondern er scheint mir eine einseitige Umbildung zu sein. Die Stufenfolge der Objektivität, darch welche der Neukantianismus den Gegensatz von Wesen und Erscheinung vermeiden möchte, hat Kant wohl beachtet, und ausdrücklich hat er jenen Unterschieden seinen eigenen Erscheinungsbegriff als den „transzendentalen" gegenübergestellt.

Nein, die einzig natïrliche Fartbildung der Kantschen Erkenntnistheorie, za der sein System von verșchiedenen Seiten ans hindrängt, liegt nicht in der idealistischen, sondern in der realistischen Richtang, und man gelangt zu ihr durch eine Revision der Bestimmungen, die Kant über das sog. Ding an sich and seine Erkennbarkeit gemacht hat. Ich freue mich, das Vorhandensein dieser Anlage in Kants Kritizismus ganz nẹerdings wieder von kompetenter Seite durch eine rein historische -Untersuchng bestätigt zu finden: in der soeben veröffentlichten Berliner Akademie-Abhandlung von B. Erdmann über die „Idee yon Kants Kritik der reinen Vernunft".

Wir gelangen m. E. zu einer allseitig befriedigenden Auffassung, die dem $Z_{\text {wiespalt }}$ von Wesen und Erscheinung die Wurzel abgräbt and ans aller Schwierigkeiten überhebt, wenn wir von Kant ansgehend zunächst einmal seinen. Exkenntnisbegriff einer Klärung unterziehen, deren er dringend bedarf. Kant hat in unkritischer Weise vorausgesetzt, daB zam Erkennen eines Objoktes in letzter Linie eine Anschanung des Objekts irgendwie not. wendig gehöre. Gleich im ersten Satze der transzendentalen Aesthetik sagt er das mit aller Deutlichkeit. Aber in Wahrheit gibt uns Anschanang überhaupt keine Erkenntnis; sie ist ganz unwesentlich dabei. Sie lehrt uns wohl Gegenstände kennen, niemals aber erkennen. Die Verkennung dieses Unterschiedes, den ich bei früheren Gelegenheiten oft schon zu betonen hatte ${ }^{\mathrm{X}}$ ), ist die Ursache der gefährlichsten Irrtümer in der Philosophie ge-

1) Vgl. z.B. meinen Aufsatz: Gibt es gine intuitive Erkenntnis? Viertel jahrsstchr. f. wiss. Phil., Bd. 36. 
wesen, gerade auch mancher neueren Systeme, die auf dem Wege reiner Intuition oder Wesensschan wissenschaftliche Erkenntnis zü erringen hoffen.

Beí sorgfälltigster Analyse aber.ergibt sich: Einen Gegenstand oder V̈organg er.kenn en heißt ị̣mer: andere Gegenstânde oder Vorgänge in Îhm aufinden, solche Beziehnngen zwischen ihm and ihnen entdecken, daß er durch sie dargestellt und auf sie zurückgeführt werden kann. Er wird auf sie zarüïkgefuhrt, das heibt: ér wird áls Spêzialfall von allgemeinen Fällen dargestellt. Es bedarf nun diesem Prozeb keiner unmittelbaren Anschanung der Gegenstände, soñdern es genügt, wenn wir imstande sind, sie dareh Begriffe zu bézèichnen. Daza ist weiter nịchts nötig als eine eindeatige Zuordining der Bégriffe zu den Ofjekten. Und Eirkenntnis bedeatet demnach die Schaffung einer Ordnung zwischen diesen

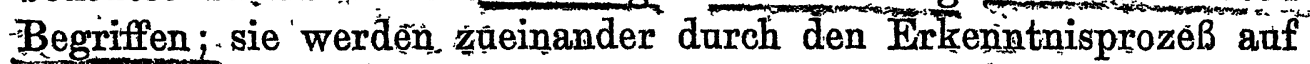
die beschriebene Weise in Beziehung gesetzt, und damit sind die Objekte erkănnt, denen jene Begriffe zugeordnet wareñ; dèr Ordnüng der Objekte entsprieht die Beziehung der Begriffe zủeinander. Erkenntnis bedeatet also Stiftung einer Beziehung zwischen den za erlennenden Objéktén - oder vielmehr zwischen ibren Begriffen, incht aber stellt sie eine reale Beziehnng her zwischen dem errkennenden Subjekt und den erkannten Gegenständen. Im Gegensaitz dazu bedeutet Anschauing gerade einen innigen realen Konnex zwischen Anschauendem und Angeschauten. Bei einem anschavlichen Erlebnis - wenn ích z. B. einen Ton höre - tritt dieser Ton tatsächlich in Realunion mit den andern Erlebnissen, die mein Ich konstitûieren; er gehört zu diesem Ich, er wird vơn mair erlebt, er wird mir dadarch bekannt. Aber erkannt wird er dadurch noch-keineswegs; dazu gehört noch ganz etwas anderes; daza müBte sein Begriff $z a$ andern Begriffen in Beziehnng gesetzt, müBten z. B. die Bedingnngen seiner Entstehung erforscht werden, und dadurch wäre er in den allgemeinen Zusammenhang der Natur einziordnen, mit Hilfe des Begriffs der. Schwingungszahl and anderer. Einen direkteren Weg zu seiner Erkenntnis gibt es nicht; đadürch, ' $\mathrm{da}$ ich ihn nür höre, anschaulich erlebe, wird er bloß ge g e ben, nicht erkannt. Und umgekebrt: um etwas zu erkennen, brauche ich es keineswegs unmittelbar anzuschanen; sondern ick. brauche nur die Beziehnngen aufzusuchen, in denen der Begriff davon zu andern Begriffen steht. Das folgt ganz allgemein aus der Analyse des Erkenntnisbegriffs; and wenn es daher überhaupt 
eine Wesens erkenntnis geben soll, so kann sie nicht Wesens schau sein, sondern eben auch nur eine Beziehung and Ordnung der dem Wesen zugeordneten Begriffe. Ein Blinder, der nie einen Lichtschimmer geschaut hat, kann doch das W és en des Lichts vollkommen erkennen, nämlich durch das Begriffssystem der theo" No retischen Optik. Nar kennen, er leben kann er es nie - das ist eben etwas anderes, hat mit Erkenntnis nichts za tan. Dem Phÿsiker ist das Wesen-der Elektrizität allein beschlossen in den Gleichungen Maxwells, das Wesen der Gravitation in den Gleichungen Einsteins; er denkt nicht daran, es schauen za wollen.

Doch jetzt die Anwendūng auf ûnser Problèm. Nur solange man das unmittelbare Anschauen für eịne notwendige Bedingung der Erkenntnis hält, fällt der Gegensatz von Erscheinung und̆ Ding an sich mit dem des Erkeñnbaren und des Unerkennbaren zusammen. Denn anschaulich sein heißt Bewaßtseinsinhalt sein, and das sind freilich nur die "Erscheinungen" - die Dinge an sich aber sind ihrer Definition nach nicht Bewußtseinsinhalt und folglich der Anschauung gänzlich entzogen. Wir wissen aber jetzt, $\mathrm{da} \hat{B}$ dies' kein Hindernis ihrer Erkenntnis za. sein braucht könnten wir sie anschauen, so würde uns das zu ihrer Frikenntnis unmittelbar gar nichts helfen. Wenn wir überhaupt Gründe haben; außerhalb der Bewuftseinswirklichkeit noch reales Sein anzunehmen, so ist es aụch erkennbar. Denn dazu wird nur vorausgesetzt, $\mathrm{da} B$ jenen Realitäten irgendwie Begriffe als Zeichen eindeutig $z \mathfrak{z}$ geordnet werden kồnnen - eine solche Zuordnang jedoch wird von einzelwissenschaftlichen, empirischen Gesichtspunkten aus ebenso. gebieterisch and mit denselben Gründen gefordert wie das Dasein jener Realitäten selbst. Dièse aber nun als "Dinge an sich“ zu bezeichnen, haben wir gar keinen Anlab mehr, denn erstens werden wir uns vor dem Worte „Ding “ hüten, weil darin manche Nebengedanken liegen (z. B. der der Substantialität), die wir vermeiden müssen, and anßerdem sind-sie dadurch, daB wir sie in dem Netz der Begriffe einfangen, aus Wirklichem an sich zugleich zu Wirklichem für uns geworden. Ferner ist nun aber auch der Terminus „Erscheinung" für das Reich des Bewaßtseinswirklichen nicht mehr passend, denn es lassen sich die Merkmale gar nicht mehr an ihr finden, die für den Erscheinangsbegriff durch die ganze Entwicklung der Philosophie hindurch gerade die wesentlichen waren.

So schwindet der Gegensatz von Wesen und Erscheinung ganz 
von selbst. Damit wir dies deutlich einsehen und ein klares Bild . on dem Standpunkt gewinnen, za. dem wir uns auf diese Weise. érheben, viergegenwärtígen wir uns noch einmal die genaue Bedeutung unseres Begriffspaars, die durch alle besprochenen Wāndlangen hindurch dieselbe blieb und:eben das ganze Pröblem in sich schliebt.

Das Wort Erscheinung deutet stets hin auf etwas außerhàlb Liegendes, das da erscheint, and ohne welches die Erscheinung nicht da wäre. Dagegen könnnen die wesenhaftén Realitäten, màğ man sie als Dinge oder sonștwie fassen, sehr wöhl vorhanden sein, ohn'e irgend einem Subjekte zuu erscheieinen. Sie sind also der Erscheinung gegenübịer etwas Selbständiges, Ũnabhängiges; es bestèt zwischien beiden Glièdèn ène einseitige Abhängigkeit, welche die Erscheiñungen jener Selbständ̈igkeit beraubt, die zu dem Begriffe 'des' wesenhät Wirklichen unabtrennbar gehört. -

Es gibt keine Tatsäche, die zu" einer derártigen Gegenüberstellung zweier irređuzibler Realitäten zwänge oder auch nur berechtigte, von denen die eine ganz auf sich selbst ruht, während díle andire von ihr ábhängig ist. - Wir gelangen zu einem befriedịgenden Weltbilde nur dann, wenn wir allem Wirklichen, den Bewaßtseinsinhalten sowohl wie allem anBerbewaBten Sein die gleiche Art und den gleichen Grad von Realität ohne jeden Unterschied zuerkennen. "Alle sind im. gleichen Sinne selbständig, alle aber auch in glêfchem Sinne voneinander abhängig. Das heißt: Die Vorgänge in meinem Bewaßtsein werden nicht nar durch eine transzendente W'elt beding̈, deren Erscheinungen sie wären, sondern sie stehen vollkommen gleich wirklich und gleichberechtigt neben jener außerbewaßten Welt und 'bedingen ihrerseits die Vor-

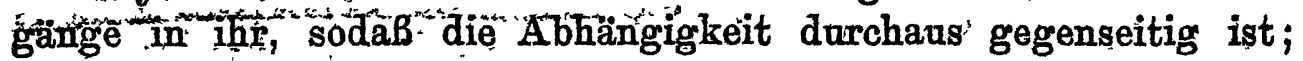
und es fehlt jeder Grund, anzanehmen, daB die Wechselbeziehangen žwischen deñ beiden Reichen anderer Natur wären als die innerhálib eines jeden der beiden bestehenden. Es sind also überhaupt gar nicht zWei Reiche verschiedener Art, sondern nur Teile eines éinzigen Wirklichleêtsreiches, von denen der eine zufällig za unserm Bewaßtsein gehört, der andere nicht. Der eine ist anschanHich gegeben, der andere zufällig nicht. Deswegen ist aber der letztere nicht etwa weniger gut erkenubar als der erste - im Gegenteil, die Physik ist bisher unvergleichlich erfolgreicher gewesen als die Psychologie. Die Anschanlichkeit der psychologischen Gegenstände nütżt eben nichts für ihre Erkenntnis, sondern 
es kommt allein auf die Möglichkeit der begrifflichen Verarbẹ̦itang and Ordnung an. Darch das Begriffssystem der Naturwissenschaften erkennen wir tatsächlich das W e sen der extramentalen Wirklichkeit. Nicht uner kennbar ist sie, wie der Phä̉nomenalismus seit Kant immer wieder behauptet, sondern nur unken nbar. anerlebbar, and das ist etwas ganz anderes. Die Bewaßtseinswirklichkeit ist ein Gefüge zahlloser unaufhörlich Wechselnder Qualitäten; and wenn man uns fragt, was denn nun die übrige reale Welt sei, so werden wir antworten: nun, sie ist eben anch ein Gewebe unablässig wechselnder Qualitäten. Die weitêre Frage, wiè denn nun diese Qualitäten an sich beschaffen seien, $d . h$. unabhängig von unserer Beżeichnüng durch Begriffe, wird als falsch gestellt abgelehnt werden "müssen, denn jede nur denkbare Antwort auf solch eine Frage könnte eben doch wieder nichts andres leisten and bedeuten als eine Bezeichnung durch einen Begriff: Was sich über das Wesen der AnBenwelt sagen läßt, ist vollständig enthalten in dem ihr zugeordneten Begriffssystem, dàs wir. Naturwissenschaft nennen. Wer aber damit nicht zufrieden ist, sondern etwa begehrt, die extramentalen Qualitäten kennen zu lernen, so wie er die eigenen mentalen Qualitäten kennt - z. B. Last, Schmerz, grïn, warm -, aus dem spricht nicht der Wille zor Erkenntnis, sondern der Wille zum Erleben, zqu Schanen. Was er wünscht, hat mit Erkenntnis nichts zu tun. Wir haben keinen Grand, über die Unerkennbarkeit der Welt zu klagen, and darüber, daß etwa nar ihre „Erscheinung" uns zagänglich sei. Im. Gegenteil, was wir durch nnsere Begriffe erkennen, ist immer nur Wesen; es gibt keine Erscheinung.

Die unmittelbarep Daten des Bewabtseins sind selbständiges Sein, vollgehaltiges Wesen; and wir vermögen keinen Sinn mehr zu verbinden mit der Behauptang, daß sie nur die Phänomenè eines verborgenen, transzendenten Seins wären. Gewiß sind sie von andern Realitäten abhängig, aber nur in dem Sinne, in dem oben überhaupt alles Wirkliche miteinander zusammenhängt - und wenn jemapd das Erscheinang nennen wollte, so wäre alles Erscheinung von allem. Unsere Erlebnisse, Wahrnehmungen, Vorstellangen und Gefühle sind nicht etwas_Sekundäres, nicht ein Soin zweiten Grades, sondern in dem gleichen Sinne selbständig real wie irgend welche transzendenten Wesen. Es gibt nur eine Wirklichkeit, and sie ist immer W es en. Gewiß gibt es unendlich viele Ártèn wirklicher Gegenstände, aber es gibt nur eine Art der 
Wirklichkeit, and sie kommt ihnen allen in gleicher Weise zu. Das war eben der Grundfehler, der ïberall Anlaß zur Entstehnng des Problems, ja fast zu jedèm phịlosọphịschen Dualismus gab: man: glaabte an verschiedene Arten von Realität, wo man nur von verschiedenen Arten des Realen hätte sprechen dürfen. Da man nun merkte, daß zwei Reálitäten sịch miteinander nicht vertragen, so mußte man eine von ihnen aus der Welt schaffen. Die alten Philossophen versuchten das, indem sie die Erscheinangen für nnwirklich ,erkilärtén, die neneren suchten statt dessen lieber die sog. Dinge an sich losźw werden. Beide haben unrecht, weil sie mit der anfänglichen Zerlegung der Welt in Wesen ựd Erscheithing anrecht hatten.

Nar wenn wir̆ jede Trennang innerhalb des Wirklichkeits= begriffs vermeiden̆, bleiben wir seinem ursprünglichen Sinne ge= treu. Seine.Quelle war das unmittelbar Gegebene, dièses ist schlecht= hin real, und die Fragesstellung des Realitätsproblems richtet sich darauf, ob di d s s lb o Realität anßerdem noch andern Gegenständen zugeschrieben wërdèn darf. Wer die Wirklichkeit der letzteren àls . etwas anderśartiges and Neũes betraçhtet, nimmt jenem Problem aèn Sinn and erfindèt frei einẹn Realitätsbegriff, der der Erfahruigsgrandlage entbehrt and in endlose Widersprüche führt.

Es gibt nur e ine. Wirklichkeit, and alles, waslin ihren Bereich fầilt, ist unserer Erkenntnis prinžipiell auf gleiche Weise zugänglich, dem Daisein wie dem Wesen nach. Nur ein kleiner Teil dieser Wirklichkeit ist uns jeweils gegeben, alles übrige ist uns nicht gegeben, aber die dadurch bedingte Scheidung des Erlebten und des Nichterlebten, des Subjektiven und Objektiven ist zufalliger Art, nicht prinzipieller Natur, wie es diejenige zwischen Wesen und Erscheinung sein sollte, die wir als unmöglich erkannt haben.

". 'Ist diese Einsịcht richtig, wie ich zaversichtlich glaube, so würde dảmit nicht bloß die Fragé nach dem Verhältnis von Wesen rnd Erścheinung verstammen müssen, sondern wegen der eingangs tiervorgehobenén innigen Verflechtung der philosophischen Probleme wäre auch aüf añdere, noch tiefergreifende Fragen ein klärendes Iicht geworfen, and ein weiteres Stück Weges zur Einheit des philosophischen Weltbildes läge in hellerer Beleuchtang vor uns: 